\title{
AVALIAÇÃO DOS ÍNDICES DE MORTALIDADE POR DOENÇAS CARDÍACAS E RESPIRATÓRIAS NA MACRORREGIÃO DO JEQUITINHONHA-MG
}

\section{Jéssica Stéfany Rocha ${ }^{1}$; Gabriela Cristina Rosa ${ }^{1}$; Cíntia Maria Rodrigues ${ }^{2}$; Thiago Ferreira Santos $^{3}$; Maylza de Fátima do Nascimento ${ }^{3}$; Heloisa Helena Barroso ${ }^{4}$; Ana Paula Nogueira Nunes $^{5}$}

${ }^{1}$ Graduanda em Fisioterapia. Universidade Federal dos Vales do Jequitinhonha e Mucuri (UFVJM), Diamantina, MG.

${ }^{2}$ Enfermeira. Doutoranda em Enfermagem Fundamental pela Escola de Enfermagem Fundamental de Ribeirão Preto (EERP) da Universidade de São Paulo (USP), Ribeirão Preto, SP.

${ }^{3}$ Graduando em Nutrição. Universidade Federal dos Vales do Jequitinhonha e Mucuri (UFVJM), Diamantina, MG.

${ }^{4}$ Enfermeira. Mestre em Ensino em Saúde pela Universidade Federal dos Vales do Jequitinhonha e Mucuri (UFVJM), Diamantina, MG.

${ }^{5}$ Doutora em Saúde Coletiva/Epidemiologia pela Universidade do Estado do Rio de Janeiro (UERJ). Docente na Universidade Federal dos Vales do Jequitinhonha e Mucuri (UFVJM), Diamantina, MG.

\section{DOI: 10.47094/IICNNESP.2021/29}

\section{RESUMO}

As doenças cardíacas e respiratórias, caracterizadas no CID:10 do sistema DATASUS apresentam impacto direto na qualidade de vida da população. Atrelado a isso, essas doenças correspondem as maiores causas de morbimortalidade no Brasil. Para tanto, esse trabalho objetivou investigar a mortalidade geral na macrorregião Jequitinhonha - MG para doenças cardíacas e respiratórias. Tratase de um estudo ecológico descritivo, com análise parcial dos dados de mortalidade geral da região do Jequitinhonha - MG, avaliando os dados secundários do Sistema de Informações sobre Mortalidade - SIM, no DATASUS, nos últimos 11 anos, a partir de 2009. Como resultado, observa-se que dentre as causas de mortalidade estudada, as doenças do aparelho circulatório são as que mais têm causado óbitos e em segundo plano as doenças respiratórias que estão em ascenção. Os achados evidenciam a importância da abordagem desta problemática, para orientar e traçar um perfil sobre o que vem ocorrendo com a população.

PALAVRAS-CHAVE: Epidemiologia. Saúde Pública. Doenças Crônicas.

ÁREA TEMÁTICA: Condições Sociais e de Saúde 


\section{INTRODUÇÃO}

As doenças cardíacas e respiratórias, caracterizadas no CID:10 do sistema DATASUS apresentam impacto direto na qualidade de vida, da população. Essas patologias trazem limitações e influenciam no desempenho das atividades de vida diárias. Atrelado a isso, tem-se que essas doenças correspondem as maiores causas de morbimortalidade no Brasil. Contudo, não existem dados que acompanham a evolução nos últimos 11 anos dessas doenças relacionadas na macrorregião do Jequitinhonha - MG. Para tanto o objetivo desse trabalho é investigar a mortalidade geral na macrorregião Jequitinhonha - MG para doenças cardíacas e respiratórias. (COSTA, N. A; COSTA, M.C.P., 2020).

\section{METODOLOGIA}

Trata-se de um estudo ecológico descritivo, com análise parcial dos dados de mortalidade geral da região do Jequitinhonha - MG. O estudo avaliou os dados secundários do Sistema de Informações sobre Mortalidade - SIM, no DATASUS, para essa região dos últimos 11 anos, a partir de 2009. Foram abordados então as doenças cardíacas e respiratórias, classificadas no CID:10.

A análise dos dados, consistiu em exportação de uma tabela no formato Excel, com as informações sobre mortalidade, analisando-se a média aritmética e a porcentagem dos dados brutos obtidos.

\section{RESULTADOS E DISCUSSÕES}

O estudo realizou uma comparação da série de dados brutos de mortalidade por causa respiratória e cardiovascular durante o período de 2009 à 2019. Os resultados preliminares desse estudo, são evidenciados na tabela e quadro abaixo.

Tabela 1: Óbitos totais na macrorregião do Jequitinhonha - MG causados por doenças do aparelho circulatório e doenças do aparelho respiratório (2009-2019).

\begin{tabular}{|c|c|c|c|c|c|c|c|c|c|c|c|c|}
\hline Taxas de Mortalidade & ANO & & & & & & & & & & Total \\
& 2009 & 2010 & 2011 & 2012 & 2013 & 2014 & 2015 & 2016 & 2017 & 2018 & 2019 & \\
\hline Doenças Cardíacas & 481 & 534 & 593 & 573 & 536 & 503 & 521 & 541 & 575 & 535 & 561 & 5953 \\
\hline Doenças respiratórias & 167 & 183 & 198 & 210 & 168 & 227 & 244 & 250 & 264 & 244 & 269 & 2424 \\
\hline TOTAL & 648 & 717 & 791 & 783 & 704 & 730 & 765 & 791 & 839 & 779 & 830 & 8377 \\
\hline
\end{tabular}

Fonte: MS/SVS/CGIAE - Sistema de Informações sobre Mortalidade - SIM. 
Gráfico 1: Óbitos por ano de ocorrência de 2009 a 2019 no Vale do Jequitinhonha (n= 8377) causados por doenças do aparelho circulatório e do aparelho respiratório

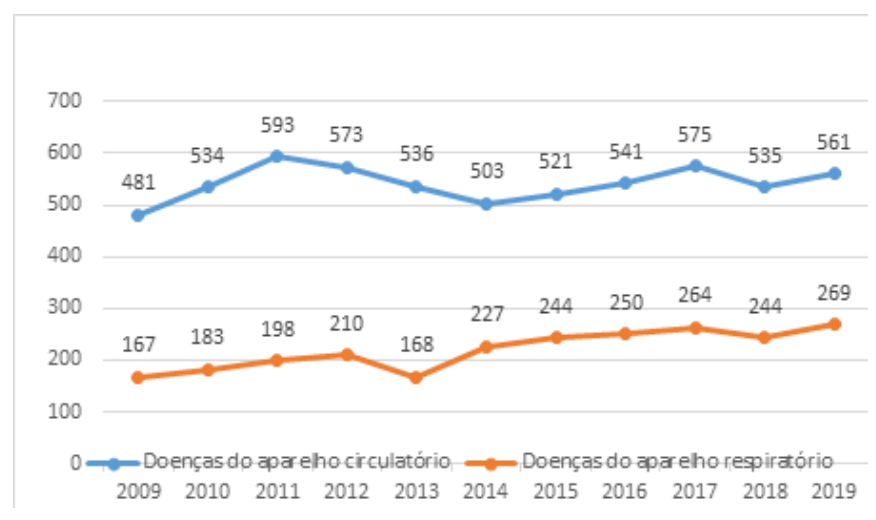

Fonte: Elaboração dos autores (2021).

Observa-se que dentre as causas de mortalidade estudada, as doenças do aparelho circulatório são as que mais têm causado óbitos pelo Vale do Jequitinhonha no período de onze anos entre 2009 e 2019, tendo o seu ápice no ano de 2011 com 593 mortes registradas e a menor incidência em 2009 com 481 mortes. Estes onze anos registram uma média aritmética de 541,18 óbitos.

A curva de incidência de óbitos por doenças do aparelho respiratório se encontra de forma ascendente excetuando os anos de 2013 e 2018, onde em 2013 houve uma baixa parecida ao ano de 2009 , porém volta a crescer de forma abrupta no ano seguinte. Nesse espaço de tempo, nota-se que o aumento de mortes por doenças respiratórias foi de 161,08\%.

Entre os principais resultados destacam-se o elevado número e a tendência de crescimento dos óbitos, em destaque, os óbitos por doenças circulatórias. Os dados apresentados refletem a soberania das doenças crônicas como causas de morte, tendo as disfunções do aparelho circulatório uma proporção de mais impacto, evidenciando a transição epidemiológica. (CARVALHO, M. H. R; CARVALHO, S. M. R; LAURENTI, R.,2014).

Assim as doenças do sistema circulatório são apresentadas neste estudo, em primeiro lugar como primeira causa de óbitos na população da macroregião do Jequitinhonha também corrobora com o estudo realizado por Piuvezam e cols., que evidencia mortalidade por doenças cardiovasculares em idosos no Brasil em 2015 (PIUVEZAM et al., 2015).

Em relação às doenças respiratórias, houve um leve crescimento da proporção de óbitos na última década. Os óbitos por doenças respiratórias ainda são prevalentes devido principalmente ao crescimento e envelhecimento populacional, possivelmente associado à transição epidemiológica para doenças não transmissíveis (LEAL et al., 2020).

As próximas etapas de investigação do estudo pretendem continuar avaliando os dados brutos e as taxas relacionadas a mortalidade por doenças cardíacas e respiratórias na macrorregião do Jequitinhonha - MG através da utilização de dados secundários do Sistema de Informações sobre Mortalidade - SIM. 


\section{CONSIDERAÇÕES FINAIS}

Os achados evidenciam a importância da abordagem desta problemática, para orientar e traçar um perfil sobre o que vem ocorrendo com a população da macrorregião do Jequitinhonha- MG, em relação a mortalidade por doenças cardíacas e respiratórias.

\section{REFERÊNCIAS BIBLIOGRÁFICAS}

BRASIL, Ministério da Saúde. Banco de dados do Sistema Único de Saúde-DATASUS.

COSTA, Nayla Alves; COSTA, Magnania Cristiane Pereira; ANDRADE, Renata Aline. Internações por doenças cardiovasculares com ênfase no acidente vascular cerebral. Diamantina, Vozes do Vale, 2020.

PIUVEZAM, Grasiela et al. Mortalidade em Idosos por Doenças Cardiovasculares: Análise Comparativa de Dois Quinquênios. Arq Bras Cardiol. Natal, v.105, n.4, p.371-380, 2015.

LEAL, Lisiane Freitas et al. Epidemiology and burden of chronic respiratory diseases in Brazil from 1990 to 2017: analysis for the Global Burden of Disease 2017 Study. Rev bras epidemiol, 2020.

CARVALHO, Maria Helena Ribeiro; CARVALHO, Sebastião Marcos Ribeiro; LAURENTI, Ruy. Tendência de mortalidade de idosos por doenças crônicas no município de Marília-SP, Brasil. Epidemiol. Serv. Saúde, v.23, n.2, Apr-Jun 2014. 